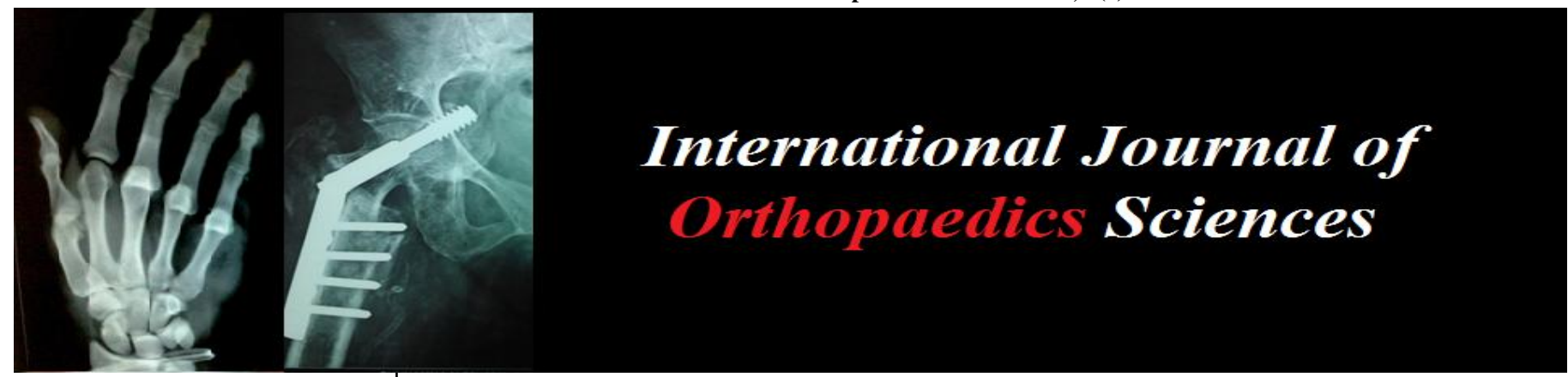

ISSN: $2395-1958$

IJOS 2018; 4(2): 95-99

(C) 2018 IJOS

www.orthopaper.com

Received: 19-02-2018

Accepted: 22-03-2018

Dr. Fardeen Sherif

M.S. Orthopaedics Assistant

Professor, Yenepoya medical

College, Mangalore, Karnataka, India

Dr. Shashikumar

M. S Orthopaedics Professor

Yenepoya Medical College,

Mangalore, Karnataka, India

Dr. Hareesha YS

Department of Orthopaedics Bangalore, Karnataka, India

Dr. Vikrant Kulkarni

Senior Resident ESIC Medical College Kalaburgi, Karnataka, India

Dr. Bhimanagouda Biradar Senior Resident Dharwad District Hospital, Dharwad,

Karnataka, India

\section{Dr. Ravishankar}

Chief Trauma Surgeon Hosmat Hospital Bangalore, Karnataka, India
Correspondence

Dr. Shashikumar

M. S Orthopaedics Professor

Yenepoya Medical College,

Mangalore, Karnataka, India

\section{The outcome of interlocking nailing of humeral shaft fractures, a one year hospital based study}

\author{
Dr. Fardeen Sheriff, Dr. Shashikumar, Dr. Hareesha YS, Dr. Vikrant \\ Kulkarni, Dr. Bhimanagouda Biradar and Dr. Ravishankar
}

DOI: https://doi.org/10.22271/ortho.2018.v4.i2b.15

\section{Abstract}

Background: The outcome of interlocking nailing of humeral shaft fractures is controversial Variations in implants, operative technique and follow-up parameters hinder comparative studies. The aim of this investigation was to evaluate the humeral nailing system according to clinical results, and to recognise advantages and disadvantages of interlocking.

Methods: A total of 30 humeral shaft fractures were treated with an Antegrade interlocking humeral nail. Results: At 12-month follow-up, bone consolidation was present in 30 cases; in 1 case complications developed. Constant shoulder score, mean Morrey elbow score, SF-12 physical score or SF-12 mental score.

Conclusion: Antegrade interlocking nailing of humeral shaft fractures can result in good functional outcome and unimpaired quality of life. Compression interlocking can minimise the fracture gap and increase the biomechanical stiffness. Potential disadvantages of compression interlocking include possible bending or loosening of the locking screw in the dynamic oblong hole.

Keywords: outcome of interlocking, humeral shaft, one year hospital

\section{Introduction}

Conservative treatment of humeral shaft fractures is well established, but has limitations in cases of nerve and extensive soft-tissue injury, multiple fractures, non-compliance or obesity. Open reduction with internal fixation is the gold standard of surgical treatment, but problems such as excessive soft tissue stripping, radial nerve injury and difficulty with complex fracture patterns are well recognised. Consequently, a wide variety of fixation devices have become available for treating these fractures, but to date there is no consensus as to the optimal method of reduction and fixation. Humeral locking nails were introduced to reproduce the success seen with similar devices used in the lower extremities. Expected advantages of interlocking nails compared with plate osteosynthesis included less soft-tissue mutilation, closed reduction and periosteum-sparing stabilisation of complex fractures. However, these theoretical advantages were not confirmed by the first clinical trials and severe complications such as shoulder pain, delayed union, non-union and iatrogenic fractures were reported. In the intervening period, progress in implant design, the possibility of antegrade insertion, improved rotational stiffness and growing experience in surgical technique have led to better results.

The aim of this prospective study was to evaluate the humeral nailing system according to clinical results, and to note the advantages and disadvantages of compression interlocking.

\section{Materials and Methods}

1year study patients with humeral fractures were treated using a humerus nail at Tertiary Hospital, Mysore, and were subsequently followed up. Of the 30 people assessed, 18 were acute fractures and 12 were nonunions, 24 were males and 6 females and one had comorbidity was on antiepilepsy treatment. The trauma mechanism was road traffic accident, including non-unions in 30 cases. The fractures were classified according to the AO system. There were two type II open fractures. In all 30 people had isolated humeral injuries.

Exclusion criteria comprised pathological fractures and fractures associated radial nerve lesions. 
Humeral fractures with radial nerve lesions were treated with nerve exploration, open reduction and plate fixation. In principle, we tried to stabilise isolated shaft fractures using a retrograde technique whenever possible. Fractures of the distal humerus shaft also indicated an antegrade technique to avoid iatrogenic fractures. Compression interlocking was generally applied to fractures with a cortical contact more than $50 \%$ of the circumference.

\section{Surgical Technique}

For the antegrade approach the patient is placed in the beach chair position. A small incision is made in line with the fibres of the deltoid muscle antereolateral to the acromion. After splitting the deltoid muscle, the supraspinatus tendon is incised in line with its fibres. The entry point close and medial to the greater tubercle is marked by fluoroscopic control, and a entry awl is used to make the entry canal. Following fracture reduction under image intensification, and reaming done, a ball-tip guide wire is advanced across the fracture site. Limited reaming is performed until cortical contact is achieved. Final reaming should be 1-1.5 mm larger than the diameter of the nail used. The ball-tip wire is exchanged for a smooth-tip wire and the nail is inserted. Proximal interlocking is performed using the target device. The distal locking screws are inserted by freehand technique into both the anteroposterior and the mediolateral nailholes.

A clinical examination and a quality of life questionnaire. Shoulder and elbow functions were evaluated using the Constant score and the Morrey score, respectively. Quality of life was analysed with the Short-Form Health Survey SF-12 questionnaire, 22 which consists of 12 questions to be answered by the patient and is divided into two sections, the SF-12 physical score (PCS-12) and the SF-12 mental score (MCS-12). The PCS-12 and the MCS-12 component summary scales are scored using norm-based methods.

\section{Materials \& Methods}

This prospective clinical study was conducted by the Department of Orthopedics of a Tertiary Hospital. We initiated this study after obtaining Institutional Ethics Committee's approval. Our study is a series of 30 cases of humeral shaft fractures treated with closed IIN over a period of 1 year.

Prospective patients were screened after obtaining written informed consent. We included adult patients of both genders with a clinical diagnosis of diaphyseal fracture of humerus, supported radiologically.

The primary objective of the study was to evaluate the functional outcome of IINF in fracture shaft of humerus. In addition, the pitfalls, complications of interlocking nailing technique, the outcome in terms of time taken for fracture consolidation, union rates were analyzed; role of early rehabilitation was also evaluate

We included patients $>19$ years with fracture of humeral shaft from $3 \mathrm{~cm}$ proximal to the olecranon fossa to $2 \mathrm{~cm}$ distal to the surgical neck of the humerus, unacceptable alignment after closed reduction of the fracture, polytrauma patients with humoral shaft fractures and closed fractures including simple, segmental, comminuted, and pathological fractures.

Patients aged <19 years were excluded as the physis is still open in them those with humeral shaft fractures involving the proximal and distal ends of the humerus and all open fractures of the humerus were also excluded.

After obtaining history, patients were assessed clinically to evaluate their general condition and local injury. Details were recorded on a preapproved proforma. Fracture site was examined for the signs of fracture. Any associated neurovascular deficit was noted.

Radiographs of the affected arm including shoulder and elbow joints were taken in anteroposterior and lateral views. Blood investigations (complete blood count, blood urea, serum creatinine, blood sugar, human immunodeficiency virus, hepatitis B surface antigen), electrocardiogram and chest Xray were taken at baseline for pre-operative evaluation.

All patients were operated under anesthesia, after a thorough evaluation, and adequate preoperative preparation. A systemic antibiotic (ceftriaxone $1 \mathrm{~g}$ ) and tetanus toxoid (intramuscularly) were given $1 \mathrm{hr}$ before surgery.

Postoperatively, the arm was placed in a sling. Early range of motion exercises for shoulder and elbow were started as soon as operative pain subsided. Emphasis was given to shoulder and elbow range of motion exercise to avoid joint stiffness. In patients without any associated injuries or the injuries did not warrant hospital stay, patients were discharged on 4th or 5th post-operative day, after instructing about the range of motion exercises. Sutures were removed on 14th post-operative day.

\section{Follow-up}

All patients were followed up at monthly intervals for 6-12 months or till the union of fracture. Importance was given to restoration of range of movements of shoulder, elbow and subjective complaints throughout the follow-up period. Radiographs were taken both in anteroposterior and lateral views to check for signs of union at immediate post-operative period, months $1,3,6,9$, and 12 months.

Assessment of patients was done on the basis of clinical and radiological union, range of motion at shoulder, elbow joints and subjective complaints like pain in shoulder and elbow joints.

Functional results were graded by the criteria of Rommens et al. (Table 1) ${ }^{[5]}$. Total functional outcome of both joints (shoulder and elbow) was taken into consideration.

\section{Statistical analysis}

The data were captured on Microsoft-Excel (2007) worksheets after editing for completeness and consistency. Results were expressed as descriptive measures for continuous variables (mean and standard deviation [SD]) and as $\%$, frequency distribution for categorical variables. Subgroup analysis was carried out based on primary outcome measures. Tables and figures are used as appropriate.

\section{Results}

In this study of 1 year duration, 30 cases of humeral shaft fractures treated by intramedullary nailing were evaluated.

Study population consisted of $24(80 \%)$ males and $6(20 \%)$ females, with a mean \pm SD of $39 \pm 13.31$ years; minimum age was 21 years while maximum was 65 years.

Laborers and agriculturists $(n=8,26.6 \%)$ were most frequently affected followed by homemakers $(n=5,16.6 \%)$ (Fig. 2).

There was no significant difference between the sides affected; right side involvement was seen in 16(53.4\%) and left in 14 patients $(46.6 \%)$.

Road traffic accident was the frequent cause $(n=18,60 \%)$ and rest $(n=12,40 \%)$ had a history of fall. Indirect injury was the cause in $20(66.66 \%)$ and direct injury in $10(33.33 \%)$ patients $\&$ non unions had occurred in 6 patients.

Middle $1 / 3$ rd of shaft of humerus was fractured in $16(53.33 \%$ ) patients (Fig. 3). 
$10(33.3 \%)$ patients had oblique fracture, $10(33.3 \%)$ patients had transverse fracture, $8(26.6 \%)$ patients had comminuted fracture, and 2(6.6\%) patients had spiral fracture.

Table 2 shows the Association for the Study of Internal Fixation (AO) sub-classification.

$9(30 \%)$ patients had associated injuries; radial nerve palsy $(\mathrm{n}=03,10 \%)$ was the frequent associated injury; ipsilateral rib fracture, fracture both bone forearm on the contralateral side, ipsilateral fracture shaft of tibia, mandible fracture, blunt abdominal injury, and head injury were noted in one patient each. Mean $( \pm \mathrm{SD})$ time interval between occurrence of injury and surgery was $7.5( \pm 2.5)$ days; eighteen $(60 \%)$ patients were operated within a week of trauma while the rest were operated between 8 and 15 days.

All were treated with closed IIN in antegrade manner except $5(16.66 \%)$ cases that required open procedure to treat radial nerve palsy. The procedure was done either under general anesthesia $(n=20)$ or brachial block $(n=10)$.

Closed intramedullary nailing was performed for $25(83.4 \%$ ) patients. These nails were passed in antegrade manner and locked in static mode. $5(16.6 \%)$ patients required open reduction because of associated radial nerve palsy, which was decompressed and the fracture was fixed with interlocking nail in static mode.

Table1: Grading Of Functional Outcome

\begin{tabular}{|c|c|c|}
\hline Grade & ROM Shoulder and elbow & Subjective \\
\hline Excellent & $<10^{\circ}$ loss of ROM in direction & None \\
\hline Moderate & $10-30^{\circ}$ loss of ROM in direction & Mild \\
\hline Poor & $<30^{\circ}$ loss of ROM in direction & Moderate to severe \\
\hline
\end{tabular}

Rom: Range Of Motion

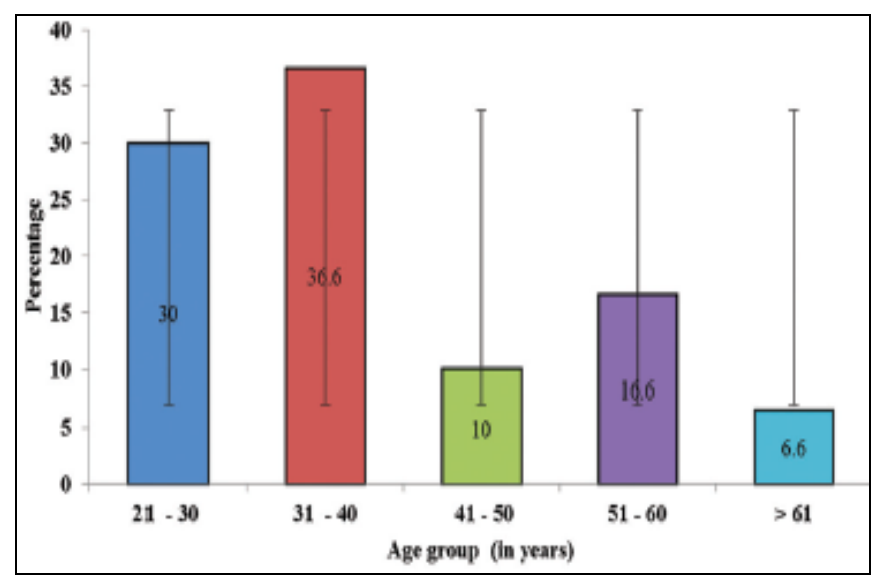

Fig 1: Age Distribution Among The Study Populatoin

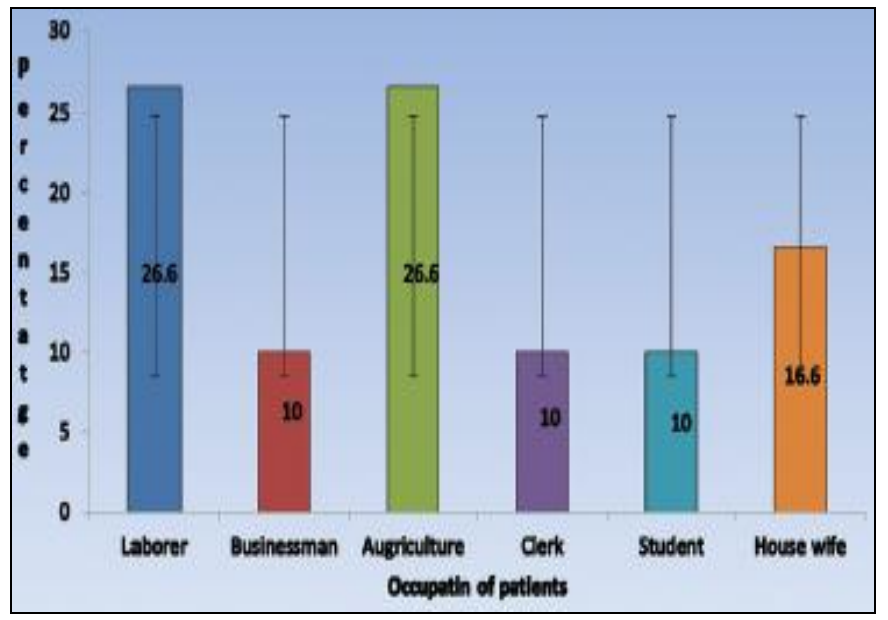

Fig 2: Occupation of study populatoin

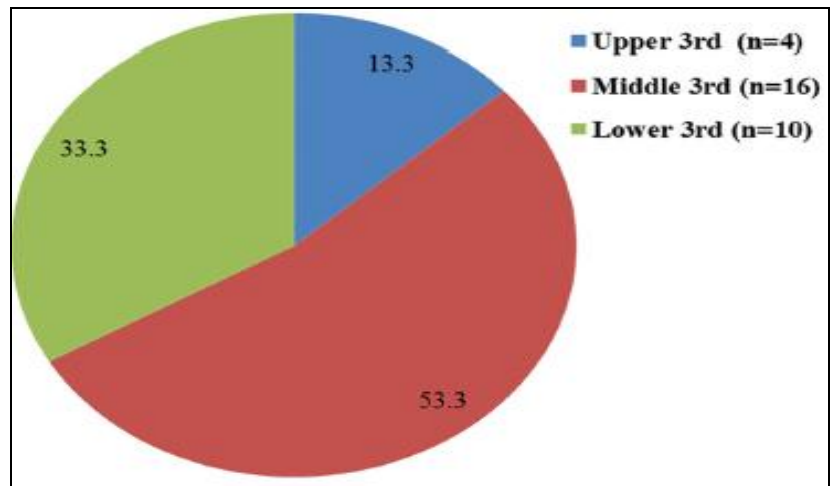

Fig 3: site of fracture of shaft of humerus in study population $(n=30)$

Table 2: Ao Sub Classification Of Injury ( $=30)$

\begin{tabular}{|c|c|}
\hline Type & Number of patients (\%) \\
\hline A & $2(6.6)$ \\
\hline A1 & $10(33.3)$ \\
\hline A2 & $10(33.3)$ \\
\hline A3 & - \\
\hline B & $4(13.3)$ \\
\hline B1 & $3(10)$ \\
\hline B2 & \\
\hline B3 & - \\
\hline C & $1(3.3)$ \\
\hline C1 & - \\
\hline C2 & \\
\hline C3 & \\
\hline
\end{tabular}

Ao: Arbeitsgemeinschaft Fur Osteoynthesefragen

Table 3: Funtioal Outcome in Study Population $(\mathrm{N}=30)$

\begin{tabular}{|c|c|c|}
\hline \multirow{2}{*}{ Grade } & $\mathbf{N}(\boldsymbol{\%})$ & \\
\cline { 2 - 3 } & Shoulder & Elbow \\
\hline Excellent & $24(80.0)$ & $27(90.0)$ \\
\hline Moderate & $5(16.67)$ & $3(10.0)$ \\
\hline Poor & $1(3.3$ & $0(0)$ \\
\hline
\end{tabular}
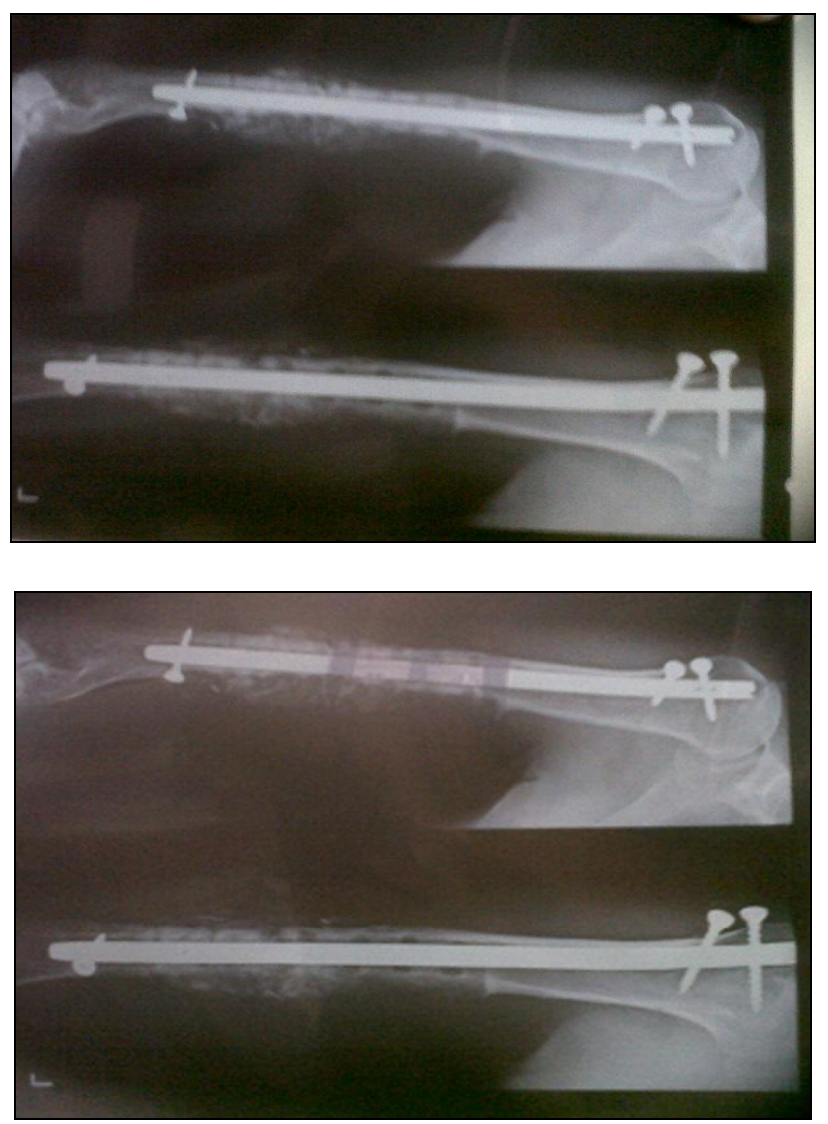


\section{Period of immobilization after surgery}

Immediate immobilization (within 3-6 days postoperatively) was advised and implemented for all, except one who was immobilized externally with POP slab for 3 weeks due to pain in the shoulder joint. The mean $( \pm \mathrm{SD})$ period for immobilization was 5.5( \pm 1.5$)$ days.

All were followed up post-surgery. The average period of follow-up was 9 months, for a minimum of 6 months, and maximum of 12 months.

The period of fracture union ranged from 10 to 16 weeks with an average period of 13-week. Nonunion was observed in one patient.

Overall functional results were excellent in $80 \%$ patients, moderate in $16.6 \%$ patients, and poor in $3.3 \%$ patients (Table 3).

Functional outcome was satisfactory in 24(80\%) patients. Restriction of joint motion was seen in patients who were immobilized for long duration $(n=05,16.66 \%)$.

\section{Complications}

There were no pre- or intra-operative complications. Although $3(10 \%)$ patients suffered additional comminution at fracture site during nail insertion intraoperatively, but did not affect the fracture union.

\section{Post-operative complication}

One $(3.3 \%)$ patient developed superficial infection which subsided with appropriate antibiotics. There was no incidence of deep-seated infection in any. Three (10\%) patients had nail impingement of proximal end due to incomplete burying into the bone. They had occasional pain in the shoulder with restriction of terminal $15-20^{\circ}$ of abduction. They had moderate functional outcome. Shoulder stiffness was observed in one $(3.3 \%)$ patient in whom abduction was affected with a range of $0-90^{\circ}$. This patient complained of severe pain, the cause was unknown. He had poor functional outcome.

Nonunion was seen in one $(3.3 \%)$ patient who was fixed in distraction at the fracture site. On follow-up, there were no signs of fracture union. The fracture ended in nonunion. A secondary procedure with autologous bone graft was performed after 9 months, which achieved union later.

\section{Discussion}

Conservative management of fractures of shaft humerus often yield satisfactory therapeutic outcome, operative stabilization is required in few including those with unsatisfactory closed reduction and multiple injuries. Moreover, surgery is preferred by orthopedic surgeons in these patients and also by patients for the rapid relief of symptoms, restoration of joint functions. Invention of newer techniques has made the surgery easier to perform with lesser complications. Plate osteosynthesis has yielded high success rate but requires extensive dissection with the risk of radial nerve damage and refracture after implant removal. Advantages of intramedullary nailing have made it the choice of surgical treatment ${ }^{[6]}$, but the use of unlocked flexible nails has been complicated by poor rotational stability and slipping of the nails causing joint irritation. Locked nailing overcomes these deficiencies, and results in satisfactory therapeutic outcome. It has been considered the treatment of choice in humeral shaft fractures in the recent past ${ }^{[7]}$. Thus, we evaluated the clinical outcome and advantages of intramedullary nailing in our patients with FSH. In our study of 30 patients, $80 \%$ were men and mean age was 39 years; laborers and agriculturists $(n=8$,
$26.6 \%$ were most frequently affected followed by homemakers $(n=5,16.6 \%)$. All these are attributable to the higher physical activity in these patients. Males are more prone to have humeral shaft fractures but few studies did not find any significant gender difference. Mean age ranged between 32 and 48 years. Road traffic accident (RTA) was the most common mode of injury in most of the studies. RTA $(n=18)$ was the cause in 16 men and mean age was 39 years (21-65 years) in these patients. This could be attributed to the increased use of two wheelers for transportation by the population in this age group. Middle-third of the shaft is the most common location of the fracture ${ }^{[5,8,9]}$ which was seen in our patients too $(53.3 \%)$. We noted that the right extremity was affected in $53.33 \%$ patients. Reports available have documented humeral shaft fractures of category A of $\mathrm{AO}$ classification system. In our study, $73.3 \%$ fractures were of category A of AO classification system. Associated multiple injuries are frequent with $\mathrm{FSH}^{[5,8,9]}$, indicating the degree of trauma in these patients. Our patients $(n=09,30 \%)$ too had associated multiple injuries. Radial nerve injury $(n=03,10 \%)$ was the most common injury reported in our patients. Other injuries included ipsilateral rib fracture, fracture both bone forearm on the contralateral side, ipsilateral fracture shaft of tibia, mandible fracture, blunt abdominal injury, and head injury.

Marty et al. Reported fracture of middle 3rd of humerus was common $(85.25 \%)$ in their study population. Simple transverse fracture of A3 type was frequent (47.54\%). Radial nerve palsy was seen in $14.75 \%$ before surgery and one patient developed nerve palsy, postoperatively. There was no incidence of infection. Functional outcome was good in $85.42 \%$. Nonunion was the complication seen in $7.14 \%$.

Most of the operative methods for stabilization of humeral shaft fractures have acceptable rates of union. Reported rates of fracture end union have been $>90 \%, 96-97 \%$ depending on the techniques and procedures followed. Nonunion of fractured ends though less, can be expected. No incidence of nonunion was reported by few studies. As the flexible intramedullary nails lack rotational control, they are frequently associated with nonunions. More rigid locked intramedullary nails have better rotational control than flexible nails, which theoretically should decrease the frequency of nonunion.

Time taken for union of fractures is clinically important following which the patient can resume normal activities. Again, there is a varied period stated by different studies. Very short period of 3.2 months have been reported by Crates and Whittle, while Lin reported 8.6 weeks. Longer time of 13.7 weeks and 18 weeks were reported by Rommens et al. And Ozturkmen et al. respectively.

In our series, $96.6 \%$ fractures united with a mean time for union of 13 weeks (range 10-16 weeks), which is comparable with the available data. We attribute early mobilization, fracture consolidation and higher union rates to nailing technique, which preserves fracture hematoma and use of unreamed nailing which preserves the endosteal vascularity promoting early fracture union.

Proximity of radial nerve to the humeral shaft makes it prone for iatrogenic injury during any operative approach to the humerus. There have been reports of iatrogenic radial nerve injury, however, with varied incidence between $0 \%$ and $3.3 \%$. There was no transient iatrogenic radial nerve palsy reported in our study.

Iatrogenic fracture comminution is frequently encountered issue in these patients; reported to be 0-3 in previous studies. 
In our study, we encountered three cases of intraoperative fracture comminution but this did not interfere with fracture healing and all these fractures united well within 4 months.

Infection is a common postsurgical complication associated with any operative procedures, more frequently with those involving humerus, because of the rich vascular supply and large soft tissue surrounding the humerus. Incidence are seen varies with the procedure adopted, higher incidence with plate intramedullary nail fixation is associated with less incidence, however, even open procedures have reported lesser incidence. Most of them occurred after open nailing and with nailing in open fractures. Incidence of infection is more with open surgeries compared to close intramedullary nailing, which may require removal of nail and appropriate antibiotic therapy.

Few reports of zero occurrence of infection have also been. Robinson et al. Reported $6.7 \%$ infection in humeral shaft fractures treated with seidel nailing. In our study, one case of the fractures got infected (superficial), which subsided with appropriate antibiotics. This we attribute to open method of nailing.

Potential deleterious effects of antegrade humeral nailing on shoulder function have been debated the most. This can be due to impingement of proximal nail tip or proximal locking screw due to adhesive capsulitis or due to rotator cuff tears. In most of the studies with antegrade nailing, 80-95\% of patients regained their normal shoulder function. In our study, $80 \%$ patients had excellent shoulder function with near normal range of motion of shoulder while $16.6 \%$ had moderately good shoulder function. These patients had impingement of proximal end of the nail. One patient had severe shoulder stiffness with significant restriction of shoulder motion.

Good therapeutic outcome considered excellent or good was obtained in $79-85 \%$ patients who were operated with the intramedullary nailing technique. Ozturkmen et al. Reported apart from excellent therapeutic outcome, moderate in $13 \%$, and poor in $8 \%$ patients. Our results are comparable to that of Ozturkmen et al. With excellent outcome in $80 \%$, moderate in $16.67 \%$, and poor in $3.3 \%$ patients.

Post-operative early mobilization of the shoulder and elbow was very critical in attaining full range of movements in these patients. The movements and the functional ability of the shoulder depend on the patient's adherence to rehabilitation program and early intensive physiotherapy hastened the recovery of shoulder function.

Closed intramedullary nailing with an interlocking nail is a safe and reliable method of treating humeral shaft fractures. Among available surgical modalities, closed nailing is the least invasive surgical technique and has the least chance of post-operative infection. It reduces the duration of the hospital stay. Complications like nonunion can be avoided by intraoperative compression and avoiding distraction at fracture site. Certain technical aspects like burying the proximal nail end at the entry portal are essential in avoiding impingement and to gain better shoulder function.

\section{Conclusion}

Closed intramedullary nailing is an excellent, least invasive surgical option available to manage humeral shaft fractures with early fracture consolidation and better union rates. It decreases the hospital stay, provides early rehabilitation and reduces the morbidity. It is ideal in patients with polytrauma and osteoporosis. Early intensive physiotherapy hastens the recovery of shoulder function.

\section{References}

1. Thakur AJ. The Elements of Fracture Fixation. (EBook). 2nd Ed. New Delhi: Elsevier India, 2007.

2. Prince EJ, Breien KM, Fehringer EV, Mormino MA. The relationship of proximal locking screws to the axillary nerve during antegrade humeral nail insertion of four commercially available implants. J Orthop Trauma. 2004; 18:585-8.

3. Garnavos C. Intramedullary nailing for humeral shaft fractures: the misunderstood poor relative. Current Orthop. 2001; 15:68-75.

4. Kolonja A, Vecsei N, Mousani M, Marlovits S, Machold W, Vecsei V. Radial nerve injury after anterograde and retrograde locked intramedullary nailing of humerus. A clinical and anatomical study. Osteo Trauma Care. 2002; 10:192-6.

5. Blyth MJG, Macleod CMB, Asante DK, Kinninmonth AWG. Iatrogenic nerve injury with the Russell-Taylor humeral nail. Injury. 2003; 34:227-8.

6. Lin J, Shen PW, Hou SM. Complications of locked nailing in humeral shaft fractures. J Trauma. 2003; 54:943-9.

7. Marchetti PG, Vicenzi G, Impallomeni C, Landi S, Surdo $\mathrm{V}$. The use of elastic nails for intramedullary fixation of humeral fractures and non-unions. Orthopedics. 2000; 23:343-7.

8. Halder SC, Chapman JA, Choudhury G, Wallace WA. Retrograde fixation of fractures of the neck and shaft of the humerus with the 'Halder humeral nail' Injury. 2001; 32:695-703.

9. Schmidt AH, Templeman DC, Grabowski CM. Antegrade intramedullary nailing of the humerus complicated by heterotopic ossification of the deltoid: a case report. J Orthop Trauma. 2001; 15:69-73.

10. Leyendecker AG, Nicolai P, Blakemore ME. Surgical emphysema formation during intramedullary reaming of a humerus. Injury. 2001; 32:74-5. 